\title{
Clinical behaviour and outcome in pediatric glioblastoma: current scenario
}

\author{
Aditya Kumar Singla ${ }^{1}$, Renu Madan ${ }^{1}$, Kirti Gupta ${ }^{2}$, Shikha Goyal', Narendra Kumar ${ }^{1}$, Sushant Kumar Sahoo ${ }^{3}$, \\ Deepak K. Uppal' ${ }^{1}$ Chirag K. Ahuja ${ }^{4}$ \\ ${ }^{1}$ Department of Radiotherapy and Oncology, Postgraduate Institute of Medical Education and Research, Chandigarh, India \\ ${ }^{2}$ Department of Histopathology, Postgraduate Institute of Medical Education and Research, Chandigarh, India \\ ${ }^{3}$ Department of Neurosurgery, Postgraduate Institute of Medical Education and Research, Chandigarh, India \\ ${ }^{4}$ Department of Radiodiagnosis, Postgraduate Institute of Medical Education and Research, Chandigarh, India
}

Received: August 16, 2020

Revised: December 18, 2020

Accepted: December 22, 2020

\section{Correspondence:}

Renu Madan

Department of Radiotherapy and

Oncology, Postgraduate Institute of

Medical Education and Research,

Chandigarh, India

Tel: +91-09868597027

E-mail: renumadan10@yahoo.com

ORCID:

https://orcid.org/0000-0002-6649-4328
Pediatric glioblastoma (pGBM) is a rare entity accounting for only approximately $3 \%$ of all childhood brain tumors. Treatment guidelines for pGBM have been extrapolated from those in adult glioblastoma. Rarity of pGBM and underrepresentation of pediatric population in major studies precludes from defining the ideal treatment protocol for these patients. Maximum safe resection is performed in most of the cases followed by postoperative radiotherapy in children over 3 years of age. Benefit of temozolomide is unclear in these patients. Here, we present the clinicopathological details and outcome of six pGBM patients treated at our institute in 2018-2019.

Keywords: Child, Glioblastoma, Genetics, Radiotherapy, Temozolomide

\section{Introduction}

Glioblastoma (GBM) is the most common primary malignant brain tumor in adults with a dismal 5 years survival of $<5 \%$ [1]. However, pediatric GBM (pGBM) is rare entity which consists of only $2 \%-$ $3 \%$ of all childhood brain tumors [1]. According to the Central Brain Tumor Registry of the United States (CBTRUS) data, the incidence of pediatric high-grade glioma ( $\mathrm{HGG}$ ) is around 0.85 per 100,000 children [2]. Owing to the rarity of pGBM, most of the studies have clubbed all HGG together without separately analysing GBM, limiting the information on its incidence, management protocol and outcome. Thus, despite the inherent difference in genetic and molecular profile, treatment guidelines for pGBM patients are indistinguishable from that in adult GBM patients. Maximum safe resection is performed wherever feasible followed by postoperative radiotherapy (PORT) along with concurrent and adjuvant temozolomide (TMZ). However, radiotherapy (RT) is usually avoided in very young children of less than 3 years of age to prevent the potential risk to developing brain.

In this paper, we aimed to describe the clinicopathological details and outcome in a series of six cases of pGBM treated at our institute (Table 1) and review the literature on this issu. All patients underwent surgery and the diagnosis was confirmed on morphology and immunohistochemistry (IHC) followed by sub-categorization of tumors using the glioma panel. As pGBM is an uncommon entity, the histopathological slides were reviewed again to reconfirm the diagnosis. 
Table 1. Clinicopathological details and outcome of pediatric glioblastoma patients

\begin{tabular}{|c|c|c|c|c|c|c|}
\hline & Case 1 & Case 2 & Case 3 & Case 4 & Case 5 & Case 6 \\
\hline Age $(y r) / S e x$ & $4 / \mathrm{M}$ & $5 / \mathrm{M}$ & $11 / \mathrm{F}$ & $15 / \mathrm{M}$ & $16 / \mathrm{F}$ & $18 / \mathrm{M}$ \\
\hline Clinical presentation & $\begin{array}{l}\text { Headache and } \\
\text { vomiting from } \\
\text { two months }\end{array}$ & $\begin{array}{l}\text { Headache and } \\
\text { vomiting from } \\
\text { one month }\end{array}$ & $\begin{array}{l}\text { Headache and } \\
\text { vomiting from } \\
\text { two months }\end{array}$ & $\begin{array}{l}\text { Headache, vomiting } \\
\text { and visual distur- } \\
\text { bances from one } \\
\text { month }\end{array}$ & $\begin{array}{l}\text { One episode of par- } \\
\text { tial seizures }\end{array}$ & $\begin{array}{l}\text { Headache, vomit- } \\
\text { ing, right sided } \\
\text { weakness from } \\
\text { one month }\end{array}$ \\
\hline Site & $\mathrm{CP}$ angle & Temporoparietal & Parieto-occipital & $\begin{array}{l}\text { Thalamus and mid- } \\
\text { brain }\end{array}$ & Fronto-temporal & Fronto-parietal \\
\hline Type of surgery & GTE & GTE & GTE & GTE & NTE & GTE \\
\hline Histopathology & GBM & Epithelioid GBM & Giant cell GBM & GBM & GBM & GBM \\
\hline \multicolumn{7}{|l|}{ Immunohistochemistry } \\
\hline $\mathrm{IDH}$ & Wild & Wild & Wild & Wild & Wild & Non-contributory \\
\hline ATRX & Lost & Lost & Lost & Lost & Lost & Lost \\
\hline p53 & Negative & Negative & Positive (mutant) & Positive & negative & Negative \\
\hline INI & Retained & Retained & Retained & Retained & Retained & Retained \\
\hline BRAF & Negative & Negative & Negative & Negative & Negative & Negative \\
\hline $\mathrm{H} 3 \mathrm{~K} 27$ & Negative & Not done & Negative & Positive & Not done & Not done \\
\hline PORT & None & $25 \mathrm{~Gy} / 5 \mathrm{fx}$ & $60 \mathrm{~Gy} / 30 \mathrm{fx}$ & $60 \mathrm{~Gy} / 30 \mathrm{fx}$ & $60 \mathrm{~Gy} / 30 \mathrm{fx}$ & $60 \mathrm{~Gy} / 30 \mathrm{fx}$ \\
\hline Concurrent TMZ & None & None & Yes & Yes & Yes & Yes \\
\hline $\begin{array}{l}\text { Number of adjuvant } \\
\text { TMZ }\end{array}$ & None & 6 cycles & 8 cycles & 6 cycles & 6 cycles & 12 cycles \\
\hline $\begin{array}{l}\text { MRI brain after } 3-4 \text { cy- } \\
\text { cles of adjuvant TMZ }\end{array}$ & $\begin{array}{l}\text { Disease progression } \\
\text { after surgery }\end{array}$ & Residual disease & Residual disease & Residual disease & Residual disease & Complete response \\
\hline Survival & $\begin{array}{l}\text { Died } 3 \text { months af- } \\
\text { ter diagnosis }\end{array}$ & $\begin{array}{l}\text { Alive at } 12 \text { months } \\
\text { of diagnosis }\end{array}$ & $\begin{array}{l}\text { Death after } 13 \\
\text { months of diag- } \\
\text { nosis }\end{array}$ & $\begin{array}{l}\text { Alive at } 15 \text { months } \\
\text { of diagnosis }\end{array}$ & $\begin{array}{l}\text { Alive at } 11 \text { months } \\
\text { of diagnosis }\end{array}$ & $\begin{array}{l}\text { Alive at } 21 \text { months } \\
\text { of diagnosis }\end{array}$ \\
\hline
\end{tabular}

GTE, gross total excision; NTE, near total excision; GBM, glioblastoma; PORT, postoperative radiotherapy; TMZ, temozolomide.

\section{Case Reports}

\section{Case 1}

A 4-year-old boy was evaluated for persistent headache and vomiting from last 2 months. Contrast enhanced magnetic resonance imaging (CEMRI) of the brain revealed a $3.9 \times 3.7 \times 4 \mathrm{~cm}$ sized space occupying lesion (SOL) in the right cerebellopontine angle for which he underwent gross total excision (GTE) of the tumor (i.e., $>90 \%$ ). The diagnosis of GBM was confirmed on histopathology and the tumor was categorised as GBM, IDH R132H-wild type; lacking $A T R X, p 53$, and $H 3 K 27 M$ mutation. A repeat magnetic resonance imaging (MRI) brain after 4 weeks revealed massive disease recurrence. Unfortunately, he could not receive adjuvant treatment due to poor performance status and expired after 3 months of surgery. Informed consent was obtained from the guardians.

\section{Case 2}

A 5-year-old boy presented with complaints of headache, nausea and vomiting for the duration of 1 month. On evaluation, CEMRI brain demonstrated heterogeneously enhancing SOL in left temporo-parietal region along with midline shift. He underwent GTE of the tumor with postoperative histopathology findings of epithelioid
GBM negative for BRAF V600E mutation. The child defaulted and presented after four months of surgery for PORT. MRI brain before PORT planning showed large residual disease and thus he received hypofractionated RT with 25 Gy in 5 fractions over 5 days by three-dimensional conformal radiotherapy (3DCRT) technique. The child responded well to the treatment and received maintenance TMZ (175 mg/m $\mathrm{m}^{2}$ on day $1-5$ every 28 days) for six cycles. A repeat CEMRI brain after third and sixth cycle of TMZ showed residual disease. Currently, after 12 months of diagnosis, he is stable and on follow-up with 3 monthly MRI.

\section{Case 3}

An 11-year-old female visited hospital for headache and vomiting of 2 months duration. On investigation, she was found to have ill-defined signal intensity in right parietal-occipital region with mid line shift on CEMRI brain. She underwent GTE of the tumor and on histology, the tumor showed features of GBM, giant cell variant that contained $p 53$ mutation; however, it was negative for IDH R132H mutation. The tumor cells revealed ATRX and p53 mutation. Microscopic appearance and IHC has been shown in Figs. 1 and 2, respectively. She received PORT with 60 Gy in 30 fractions over 6 weeks by 3DCRT technique with concomitant TMZ (75 mg/ 

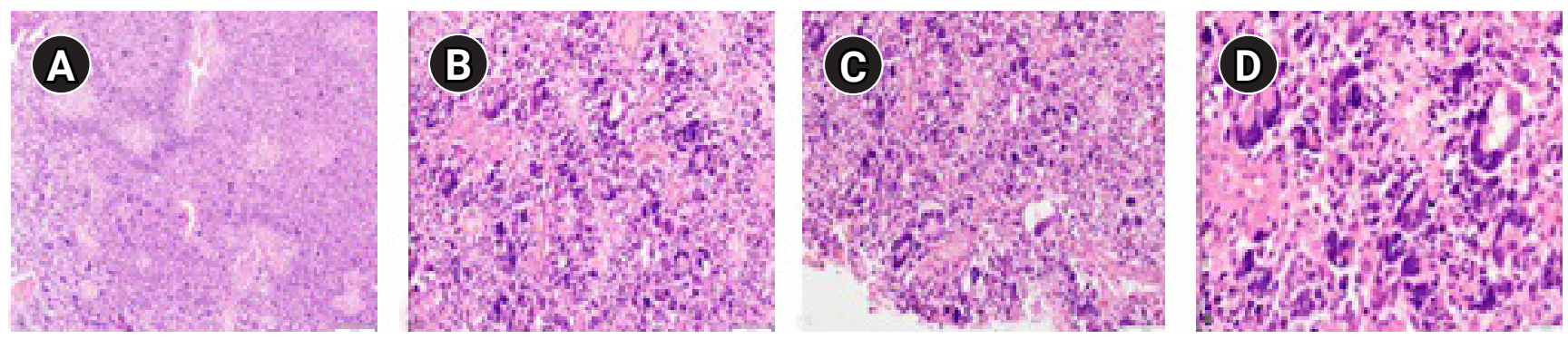

Fig. 1. (A) Low magnification showing tumor cells dispersed in sheets separated by areas of palisading necrosis $\left(H \& E_{1} \times 100\right)$. (B) Markedly pleomorphic cells dispersed in sheets against a fibrillary background (H\&E $\times 200)$. (C) Many bizarre cells including tumor giant cells are seen. Mitoses was readily identified $\left(H \& E_{1} \times 200\right)$. (D) High magnification depicting tumor giant cells with scattered apoptotic bodies (H\&E, $\left.\times 400\right)$.
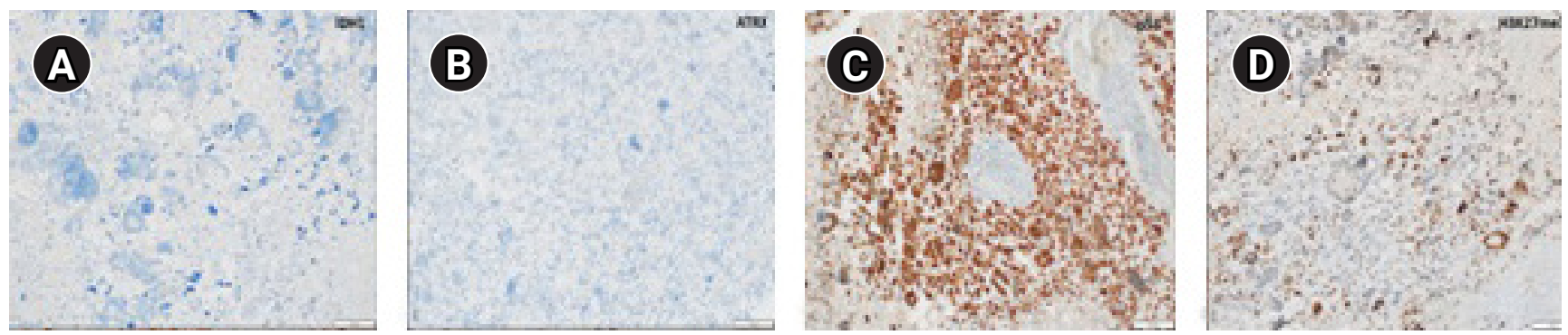

Fig. 2. (A) Tumor cells negative for IDH1 R132H mutant protein (immunoperoxidase, $\times 400$ ). (B) Tumor cells show loss of nuclear expression of ATRX (mutant phenotype) (immunoperoxidase, $\times 200$ ). (C) Strong and diffuse immunopositivity for p53 protein (mutant phenotype) (immunoperoxidase, $\times 200$ ). (D) Tumor cells show retained expression of H3K27me3 indicating absence for H2K37M mutant protein (immunoperoxidase, $\times 200$ ).

$\left.\mathrm{m}^{2}\right)$ and eight cycles of adjuvant TMZ $\left(175 \mathrm{mg} / \mathrm{m}^{2}\right)$. It was planned to continue TMZ for a total of 12 cycles; but the disease progressed and she succumbed to the disease, 13 months after the diagnosis.

\section{Case 4}

A 15-year-old child presented with complaints of headache, vomiting and visual disturbances from last 1 month. His brain MRI showed $5 \times 3.9 \times 4.9 \mathrm{~cm}$ sized mass lesion in left thalamus and lateral aspect of midbrain with perilesional oedema. He underwent craniotomy and GTE of the tumor, which, on histology was diagnosed as GBM, lacking IDH R132H and ATRX mutation, however it revealed $p 53$ mutation. Owing to the midline location, the tumor was evaluated for $\mathrm{H} 3 \mathrm{~K} 27 \mathrm{M}$ protein and was found to be positive for H3K27M mutant protein. The child received PORT with $60 \mathrm{~Gy}$ in 30 fractions over 6 weeks with concomitant and six cycles of adjuvant TMZ. Follow-up CEMRI of the brain after treatment completion showed small residual disease. Currently he is asymptomatic after 15 months of the diagnosis. Fig. 3 is showing target delineation on CT/MRI fusion at the time of radiotherapy.

\section{Case 5}

A 16-year-old girl presented with one episode of partial seizures 15 days back. CEMRI brain demonstrated $3.6 \times 3.6 \times 2.3 \mathrm{~cm}$ sized het-

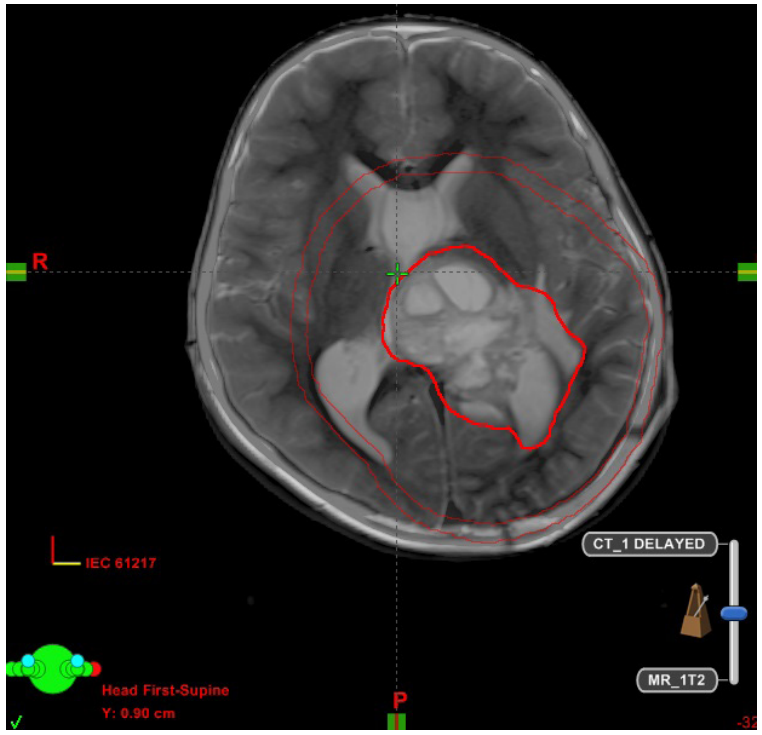

Fig. 3. CT/MRI fusion at the time of radiotherapy planning is showing large residual disease in case 4. Entire T2 signal abnormality on MRI along with post-operative cavity was included in GTV. A margin of $2 \mathrm{~cm}$ was given to GTV to create CTV. Further a margin of $0.5 \mathrm{~cm}$ was given to CTV to create planning target volume. CT, computed tomography; MRI, magnetic resonance imaging; GTV, gross tumor volume; CTV, clinical target volume. 
erogeneously enhancing tumor in fronto-parietal lobe. She underwent near total excision (NTE) of the tumor and the histopathology report confirmed the diagnosis of GBM, IDH R132H wild type lacking ATRX and p53 mutation. Following surgery, she received PORT with $60 \mathrm{~Gy}$ in 30 fractions over 6 weeks by 3DCRT technique along with concomitant and six cycles of adjuvant TMZ. Repeat MRI after treatment completion showed residual disease in the brain; however, she is asymptomatic after 11 months of diagnosis.

\section{Case 6}

An 18-year-old boy presented with headache, vomiting and progressive weakness of right side of body from past 1 month. His CEMRI brain showed a large heterogeneously enhancing tumor of size $5.6 \times 3.6 \times 4.3 \mathrm{~cm}$ in fronto-parietal lobe with midline shift. He underwent GTE of tumor and the histopathology report suggested the diagnosis of GBM lacking IDH, ATRX, p53, and BRAF mutation. He received PORT with $60 \mathrm{~Gy}$ in 30 fractions over 6 weeks by 3DCRT technique along with concomitant and 12 cycles of adjuvant TMZ. Currently he is stable after 21 months of diagnosis.

\section{Discussion and Conclusion}

GBM is a rare entity in pediatric population with actual incidence varies across the studies. Most of the published studies have combined GBM and other HGG in pediatric population together; making it difficult to assess the real incidence of pGBM. Another challenge in determining the actual incidence of pGBM is discrepancy in defining the pediatric age group that ranges from 16 to 21 years in different studies. An overall survival (OS) of 10-73 months has been reported in $\mathrm{pGBM}$ in various studies which is slightly higher than that in adult GBM patients [3]. Aetiology of pGBM is not clear and majority of the pGBM are sporadic although genetic causation has been reported in few studies $[4,5]$. It has been shown to be associated with certain syndromes like neurofibromatosis, Li-Fraumeni syndrome and Turcot syndrome [4]. pGBM patients may also harbour the matrix metalloproteinase (MMP-1) genotype [5]. Previous exposure to ionising radiation is also thought to be a causative factor.

pGBMs are commonly reported in second decade of life with a slight male preponderance, though in utero cases have also been reported [6]. In our case series, two and four patients were diagnosed in first and second decade respectively. Supratentorial brain is the commonest location for pGBM of which, cerebral hemispheres account for approximately half of the cases and frontal lobe is the most commonly involved lobe [7]. In infratentorial compartment, brainstem constitutes approximately $20 \%$ of the cases while cerebellum accounts for only $1 \%-2 \%$ of the cases of pGBM
[7]. Majority of the patients in our study (80\%) had supratentorial tumors which is in sync with the literature.

The 2016 World Health Organization classification of central nervous system (CNS) tumors has incorporated molecular phenotype into the morphology for better prognostication. IDH1 mutation forms the basis for sub-classifying gliomas into IDH1 mutant and wild-type [8]. While pGBM and adult GBM share similar histological features, they differ in genetic and epigenetic landscape such that they should be regarded as molecularly distinct entities [9]. IDH mutation is rare while mutation in F3A, H3K27M and H3.1 gene are commonly encountered in $\mathrm{pGBM}$ as compared to adult counterparts [10]. Tumors harbouring $H 3 K 27 M$ mutation arise in the midline and connotes a poor prognosis. In our series, none of the cases had IDH1 mutation ( $\mathrm{n}=0 / 6$ ). H3K27M mutation was detected in only one case. BRAF V600E was evaluated in four tumors and was not detected in any case. Studies on $0^{6}$-Methylguanine-DNA Methyltranferase (MGMT) promoter methylation have revealed little significance in $\mathrm{PGBM}$ indicating the reason behind low efficacy of TMZ [11]. MGMT promoter methylation status was not assessed in our cases.

Standard treatment guidelines are not available for pGBM cases. Results of the landmark study by Stupp et al. [12] established maximal safe resection followed by PORT along with concurrent and adjuvant TMZ as the standard of care for GBM. However, this trial included patients only in the age group of 18-70 years. Outcome in pGBM varies across the studies with 5 years survival ranging from $15 \%$ to $40 \%$ which is slightly better as compared to the adult counterpart [14-16]. Role of extent of resection (EOR) and tumor location in pGBM has been studied in few studies. In SEER analysis EOR was found to be one of the most important factors affecting outcome in pGBM patients as in adults [13]. In this analysis, a median OS of 12 months was observed with 1-, 2- and 5-year survival rates of $51.7 \%, 28.3 \%$, and $15.7 \%$, respectively. Song et al. [14] evaluated long-term outcome in 23 pGBM patients and found that median OS was significantly associated with tumor location (52 months for superficially located tumors vs. 7 months for deeply located tumors; $p=0.017$ ) and EOR (106 months for completely resected tumors vs. 11 months for incompletely resected tumors; $p<$ 0.0001). In one of the largest study, pGBM constituted approximately $1.15 \%$ of all GBM cases and approximately half of them received combined modality treatment with surgery, PORT and chemotherapy [10]. Though the median OS in pediatric patients was same as reported for adults (15 months); slightly high 5-year OS $(17 \%)$ was observed in pGBM patients. Ansari et al. [15] reported a median OS of 21.48 months after subtotal resection as compared to 33.80 months after GTE.

$\mathrm{RT}$ is integral part of the treatment in $\mathrm{PGBM}$ as in adults and a 
radiotherapy dose of 50-60 Gy has been used in various studies [16]. Trials on altered fractionated RT have not yielded successful results [17]. In contrast to adult GBM, benefit of TMZ is debatable with institutional experiences vary from adjuvant radiation alone to radiation along with $\mathrm{TMZ}$ or other alkylating agents. Mallick et al. [16] has observed favourable results for pGBM patients receiving both concurrent and adjuvant TMZ.

In our study, five out of six patients received adjuvant treatment. One patient received hypofractionated RT followed by six cycles of adjuvant TMZ while four patients received conventional fractionation RT with concurrent and 6-12 cycles of adjuvant TMZ. All patients initially responded well to the treatment. Unfortunately, one patient succumbed to the disease after 13 months of diagnosis while rest of the four patients are stable after 11, 12, 15 and 21 months of diagnosis. One child got expired even before the initiation of adjuvant treatment.

Unlike adults, the role of anti-VEGF therapy (bevacizumab) is controversial in pGBM due to infrequent expression of VEGF receptor [18]. Bevacizumab alone or with combination of irinotecan has also not proven to be effective [18]. Somatic mutation of PDGFRA (platelet-derived growth factor receptor $A$ ) has been recently reported in pGBM, hence prompting anti PDGFRA therapy which includes tyrosine kinase inhibitors like imatinib, erlotinib, gefitinib (anti-EGFR), and tipifarnib [19]. More recently poly(ADP-ribose) polymerase (PARP) inhibitors, i.e., olaparib have also shown to improve outcomes in pediatric $\mathrm{HGG}$ with $B R C A$ mutation [20].

Although rare, possibility of glioblastoma should always be considered in a child with radiological features of high grade tumor in the brain. Despite the genetic and epigenetic differences, treatment recommendations for pediatric glioblastoma patients remain the same as in adult patients leading to dismal outcome. Till date, no targeted therapies have been proven to be effective in these patients. Future research should focus towards better understanding of biology and genetics and thus to improve outcome.

\section{Conflict of Interest}

No potential conflict of interest relevant to this article was reported.

\section{References}

1. Ostrom QT, Gittleman $H_{\text {, Liao }}$, et al. CBTRUS statistical report: primary brain and central nervous system tumors diagnosed in the United States in 2007-2011. Neuro Oncol 2014;16 Suppl 4(Suppl 4):iv1-63.

2. Ostrom QT, Gittleman H, Fulop J, et al. CBTRUS statistical report: primary brain and central nervous system tumors diagnosed in the United States in 2008-2012. Neuro Oncol 2015;17 Suppl 4(Suppl 4):iv1-iv62.

3. Nikitovic M, Stanic D, Pekmezovic T, et al. Pediatric glioblastoma: a single institution experience. Childs Nerv Syst 2016;32:97-103.

4. Sgouros S, Fineron PW, Hockley AD. Cerebellar astrocytoma of childhood: long-term follow-up. Childs Nerv Syst 1995;11:89-96.

5. Malik N, Kumar R, Prasad KN, Kawal P, Srivastava A, Mahapatra AK. Association of matrix metalloproteinase-1 gene polymorphism with glioblastoma multiforme in a northern Indian population. J Neurooncol 2011;102:347-52.

6. Milano GM, Cerri C, Ferruzzi V, et al. Congenital glioblastoma. Pediatr Blood Cancer 2009;53:124-6.

7. Fangusaro J. Pediatric high-grade gliomas and diffuse intrinsic pontine gliomas. J Child Neurol 2009;24:1409-17.

8. Louis DN, Perry A, Reifenberger G, et al. The 2016 World Health Organization Classification of Tumors of the Central Nervous System: a summary. Acta Neuropathol 2016;131:803-20.

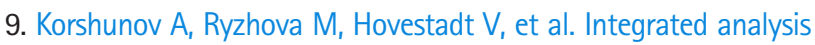
of pediatric glioblastoma reveals a subset of biologically favorable tumors with associated molecular prognostic markers. Acta Neuropathol 2015;129:669-78.

10. Liu M, Thakkar JP, Garcia CR, et al. National cancer database analysis of outcomes in pediatric glioblastoma. Cancer Med 2018;7:1151-9.

11. Artico M, Cervoni L, Celli P, Salvati M, Palma L. Supratentorial glioblastoma in children: a series of 27 surgically treated cases. Childs Nerv Syst 1993;9:7-9.

12. Stupp R, Mason WP, van den Bent MJ, et al. Radiotherapy plus concomitant and adjuvant temozolomide for glioblastoma. N Engl J Med 2005;352:987-96.

13. Adams H, Adams HH, Jackson C, Rincon-Torroella J, Jallo Gl, Quinones-Hinojosa A. Evaluating extent of resection in pediatric glioblastoma: a multiple propensity score-adjusted population-based analysis. Childs Nerv Syst 2016;32:493-503.

14. Song KS, Phi JH, Cho BK, et al. Long-term outcomes in children with glioblastoma. J Neurosurg Pediatr 2010;6:145-9.

15. Ansari M, Nasrolahi $H_{1}$ Kani AA, et al. Pediatric glioblastoma multiforme: a single-institution experience. Indian J Med Paediatr Oncol 2012;33:155-60.

16. Mallick S, Gandhi AK, Joshi NP, et al. Outcomes of pediatric glioblastoma treated with adjuvant chemoradiation with temozolomide and correlation with prognostic factors. Indian J Med Paediatr Oncol 2015:36:99-104.

17. Fallai C, Olmi P. Hyperfractionated and accelerated radiation therapy in central nervous system tumors (malignant gliomas, pediatric tumors, and brain metastases). Radiother Oncol 1997; 


\section{3:235-46.}

18. Gururangan S, Chi SN, Young Poussaint T, et al. Lack of efficacy of bevacizumab plus irinotecan in children with recurrent malignant glioma and diffuse brainstem glioma: a Pediatric Brain Tumor Consortium study. J Clin Oncol 2010;28:3069-75.

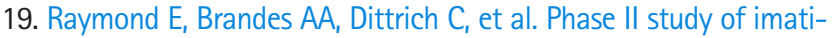
nib in patients with recurrent gliomas of various histologies: a european organisation for research and treatment of cancer brain tumor group study. J Clin Oncol 2008;26:4659-65.

20. Valiakhmetova A, Gorelyshev S, Konovalov A, et al. Treatment of pediatric glioblastoma with combination olaparib and temozolomide demonstrates 2-year durable response. Oncologist 2020; 25:e198-e202. 